\title{
MODIFIED URETEROSIGMOIDOSTOMY FOR MANAGEMENT OF MALIGNANT AND NON-MALIGNANT CONDITIONS
}

\author{
K.A. MTETA and A.Y. AL-AMMARY
}

\begin{abstract}
Objective: To investigate the outcome of Mainz Pouch II urinary diversion for both malignant and non-malignant diseases.

Design: A retrospective analysis.

Setting: Kilimanjaro Christian Medical Centre, Institute of Urology, Moshi, Tanzania from April 1995 to May 2007.

Patients: Mainz Pouch II was created in 83 patients of which, 38 were females and 45 were males (M:F 1.2:1).

Results: Early complications were seen in 11 (13.2\%) patients, as follows: one (1.2\%) prolonged ileus, $1(1.2 \%)$ wound dehiscence, two $(2.4 \%)$ perioperative deaths among the malignant group, seven $(8.4 \%)$ superficial wound sepsis. Long term complications were seen in $14(16.9 \%)$ patients, as follows: one (1.2\%) patient developed an incision hernia, one (1.2\%) patient developed unilateral pyelonephritis, one (1.2\%) patient developed unilateral ureteral stenosis, two (2.4\%) patients had deterioration of renal function, three (3.6\%) patients developed mild to moderate unilateral hydronephrosis, three (3.6\%) patients developed mucoceles. Among the 83 patients in this series, three $(3.6 \%)$ patients developed metabolic acidosis, two $(2.4 \%)$ of which, required oral bicarbonate supplementation. All (100\%) patients had daytime continence while three $(3.6 \%)$ patients had occasional night time incontinence. Overall total continence was achieved in $80(96.4 \%)$ of the patients.

Conclusion: The Mainz Pouch II is a safe and reproducible method of urinary diversion and serves as a satisfying method of continent urinary diversion in all age groups. This reconstructive surgery enabled the afflicted to achieve personal goals, hopes and aspirations, positively influencing their quality of life. The follow up show low complication rate with good results in terms of continence and quality of life, however, long term results remain to be evaluated.
\end{abstract}

\section{INTRODUCTION}

The procedure of ureterosigmoidostomy was first introduced in 1852 and quickly gained popularity during the first half of the 20th century $(1,2)$. During this early era of the procedure, unfortunately, many problems developed from the procedure and many modifications were tried unsuccessfully and the technique was abandoned for many years (3).
Ureterosigmoidostomy is undoubtedly, the oldest form of continent urinary diversion for patients born with bladder exstrophy. Ureterosigmoidostomy was first performed by Simon in 1852, and it remained the most popular method for urinary diversion until the early 1950s when the late complications of electrolyte disturbances, ureterosigmoid anastomosis site stenosis, reflux, urinary tract infections with pyelonephritis, and colon neoplasia were recognised 
and discouraged its acceptance by urologists and patients alike. The introduction and popularization of the Bricker ileal conduit to overcome these complications made urologists lose enthusiasm with ureterosigmoidostomy. Refinements in the surgical and anaesthetic techniques, improved antibiotic treatment and suture material, and the use of antireflux ureteral implantation techniques renewed the interest in that attractive and simple operation for continent urinary diversion during the 1970s. Although the postoperative mortality and morbidity have remarkably decreased, the incidence of delayed complications of pyelonephritis and hyperchloremic acidosis, as well as night-time incontinence, remained significantly high.

Recently, it became evident that most of these complications are essentially due to the unfavorable high-pressure conditions within the intact sigmoid colon and rectum. Functional studies proved that the pressure waves of physiologic bowel contractions toward the anus reach pressures exceeding $100 \mathrm{~cm} \mathrm{H} 2 \mathrm{O}$, and during defecation pressure in the intact sigmoid colon can reach up to $200 \mathrm{~cm} \mathrm{H} 2 \mathrm{O}$. Detubularisation and reconfiguration of the sigmoid colon, rectum, or both eliminate the mass contractions and high-pressure peaks and improve their dynamics to function as a compliant low-pressure reservoir (4).

In recent years, a modification of ureterosigmoidotomy using a sigma detubularised and reconfigured colon called Mainz Pouch II, was described and the technique has become an alternative procedure of urinary diversion.

Complications known to occur with Mainz Pouch II procedure are: stenosis at the ureterosigmoidal junction, metabolic acidosis requiring oral alkalizing agents, and the development of secondary malignancies at the ureterosigmoidal implantation junction $(5,6)$. The objective of this study was to present the results of the Mainz Pouch II procedure as seen in a single centre in Kilimanjaro Christian Medical Centre from 1995 to 2007, over a period of 12 years.

\section{MATERIALS AND METHODS}

This was a retrospective analysis of 83 patients who had Mainz Pouch II urinary diversion following cystectomy for both malignant bladder tumours and morphological or functional bladder loss in
Kilimanjaro Christian Medical Centre from April 1995 to May 2007. As part of the preoperative work up, the patient or the parents/guardian were counselled on the procedure and possible complications which may occur. The anal sphincter competence was judged as normal when patients could hold a 350 to $500 \mathrm{ml}$ saline enema for at least 4 to 5 hours. Anal sphincter manometry, before and after diversion was considered superfluous.

Contra-indications for the procedure were an incompetent anal sphincter, sigma diverticulosis, intestinal polyps, previous or planned radiation therapy and insufficient renal function (serum creatinine $>15 \mathrm{mg} / \mathrm{ml}$ ).

Patients were put on light and low residue diet three days before surgery and a warm soap enema was given on the night before surgery and early morning on the day of surgery. Preoperative gentamycin and metronidazole was given one hour before surgery. The surgical technique as described by Fisch et al (3) was used. At the end of the cystectomy, the lower colon lay at the bottom of the operating field with the cut ureters. The antimesenteric $20 \mathrm{~cm}$ of the lower sigmoid and upper rectum were marked with stay sutures and opened longitudinally along the taenia coli by cutting with diathermy. The posterior plate was formed by closing the posterior wall in an inverted " $U$ ", form with an inner continuous running and a second interrupted seromuscular vicryl $2 / 0$.

The ureters were implanted on the posterior plate just lateral to the midline with a $3 \mathrm{~cm}$ long submucosal antireflux tunnel, anchoring the ureters with 5 / 0 vicryl sutures. At this juncture, the apex of the pouch was anchored to the sacral promontory periosteum using a monofilament nylon suture, $1 / 0$. Both ureters were stented with a 10-F feeding tubes, which were in turn fed into a 30-F rectal tube which was then brought out through the anus and fixed to the pen-anal skin. The anterior wall was closed in two layers in similar fashion as the posterior wall. Two peritoneal drains were left in situ, abdomen closed in layers after copious peritoneal lavage. Prophylactic antibiotics were continued for three days, while patient were kept on nil by mouth for four days while the flatus tube and ureteral stents were removed on the 7th post-operative day.

During follow up patients had serum creatinine, serum electrolytes $\left(\mathrm{Na}^{+}, \mathrm{K}^{+}, \mathrm{Cl}^{-}\right)$, serum $\mathrm{HCO}_{3^{\prime}}$ ultrasound, intravenous pyelogram, investigations 
regularly. Patients were asked about continence, frequency, change of eating and dressing habits and where appropriate impact on social life and daily activities or work.

\section{RESULTS}

Between April 1995 to May 2007 in Kilimanjaro Christian Medical Centre, Institute of Urology, Moshi, Tanzania, Mainz Pouch II was created in 83 patients of which, 38 were females and 45 were males (M:F 1.2:1). Age of the subjects ranged from two months to 73 years with a mean of 34 years. Follow-up ranged from two months to 144 months with a mean of 25 months. The indication of diversion was malignant conditions in $36(43.4 \%)$ patients and $47(56.6 \%)$ nonmalignant conditions (Table 1).

Early complications were seen in $11(13.2 \%)$ patients, one $(1.2 \%)$ prolonged ileus, one $(1.2 \%)$ wound dehiscence, two $(2.4 \%)$ perioperative deaths among the malignant group, seven $(8.4 \%)$ superficial wound sepsis (Table 2).

Long term complications were seen in 14 (16.9\%) patients, one $(1.2 \%)$ patient developed an incision hernia, one $(1.2 \%)$ patient developed unilateral pyelonephritis, one $(1.2 \%)$ patient developed unilateral ureteral stenosis, two $(2.4 \%)$ patients had deterioration of renal function, three $(3.6 \%)$ patients developed mild to moderate unilateral hydronephrosis, three $(3.6 \%)$ patients developed mucoceles). Mucoceles developed exclusively among male patients with exstrophy epispadia complex where a small amount of remnant bladder tissue was left behind during cystectomy (Table 3).

Among the 83 patients in this series, three (3.6\%) patients developed metabolic acidosis, two $(2.4 \%)$ of which, required oral bicarbonate supplementation. All $83(100 \%)$ patients had daytime continence while three $(3.6 \%)$ patients had occasional night-time incontinence and these were patients who had failed obstetric vesical vaginal fistula repairs. Overall total continence was achieved in $80(96.4 \%)$ of the patients. Quality of life issues have a special significance since they determine acceptability or rejection of a surgical method in a particular community.

Quality of life is an area of study that has attracted an ever increasing amount of interest over the past two decades, particularly in the areas of health, rehabilitation, disabilities study, and social services, but also in medicine, education, and others. In health care research, the term "quality of life" has been used broadly to describe healthrelated constructs, out comes, measures, scales, and instruments. The field of quality of life research also represents a distinct scientific discipline in its own right. In addition, health related quality of life assessment has gained recognition as an important research tool for evaluating the impact of new medical treatments, procedures and health care services. The conceptual framework has three life domains, (being, belonging, and becoming) each of which has three sub domains. These domains can be scored to give a specific numerical value attributable to the quality of life.

Table 1

Malignant and non-malignant indications for Mainz Pouch II

\begin{tabular}{lrr}
\hline Indication & No. & $(\%)$ \\
\hline TB bladder & 1 & 1.2 \\
Congenital incompetent sphincter & 2 & 2.4 \\
Irreparable traumatic urethral stricture & 8 & 9.6 \\
Failed VVF repair & 15 & 18.1 \\
Bladder exstrophy epispadia & 21 & 25.3 \\
Subtotal (Non-malignant) & 47 & 56.6 \\
Neurofibromatosis bladder & 1 & 1.2 \\
Rhabdomyosarcoma bladder & 1 & 1.2 \\
TCC Bladder & 6 & 7.2 \\
SCC Bladder & 28 & 33.7 \\
Sub total (malignant) & 36 & 43.4 \\
\hline Total & 83 & 100 \\
\hline
\end{tabular}




\section{Table 2}

Post operative early and late complications seen

\begin{tabular}{lrr}
\hline Early complication & No. & $(\%)$ \\
\hline Prolonged ileus & 1 & 1.2 \\
Wound dehiscence & 1 & 1.2 \\
Perioperative deaths & 2 & 2.4 \\
Superficial wound sepsis & 7 & 8.4 \\
Subtotal & 11 & 13.2 \\
Late complication & & \\
Incision hernia & 1 & 1.2 \\
Pyelonephritis & 1 & 1.2 \\
Ureteral stenosis & 1 & 1.2 \\
Deterioration of renal function & 2 & 2.4 \\
Hydronephrosis & 3 & 3.6 \\
Mucoceles & 3 & 3.6 \\
Metabolic acidosis & 3 & 3.6 \\
Subtotal & 14 & 16.8 \\
\hline Total & 25 & 30.0 \\
\hline
\end{tabular}

Table 3

Summarised characteristics and outcomes of patients the 83 who underwent Mainz Pouch II diversion $(n=83)$

\begin{tabular}{ll}
\hline Parameter & Variable \\
\hline Age range & $2 / 12-73$ \\
Sex ratio M/F & $45 / 38(1.2: 1)$ \\
Average transfusion & 1.5 units \\
Average hospital stay & 12 days \\
Follow-up & $2-144$ (average 25 months) \\
Total incontinence & $0 \%$ \\
Night soiling & $3 / 83(3.6 \%)$ \\
Daytime continence & $83 / 83(100 \%)$ \\
Overall total continence & $80 / 83(96.4 \%)$ \\
\hline
\end{tabular}

Looking at "being: who one is" question with respect to our study one may make the following observations. Physical being: taking the example of bladder exstrophy and VVF patients who were continuously leaking urine and therefore continuously wet and smelling of urine, life styles, appearance, physical health, grooming and clothing were definitely negatively affected in the afflicted. We clearly remember a boy of four years with a continuously leaking bladder exstrophy, whom the mother was dressing with female skirt. When the mother was asked about it she responded that it was easier to change her nappies when he was in skirts rather than shorts. After urinary diversion with sigma rectum pouch, the child was seen in followup clinic dry and this time in very good shorts. To the child and the mother, this was a very important achievement in addition to the dryness and absence of continuous urine smell from the boy. Psychological being: the incontinence of urine, wetness and smell had a negative effect on the psychological health and adjustment, cognitions, feelings, self esteem, self concept and self control on the individuals whether child or adult. Imagine our VVF patients, 
who become outcasts in the society, they were always under psychological strain. Spiritual being: personal vales, personal standards of conduct and spiritual beliefs were adversely affected. A number of our patients could not participate in religious gatherings on account of wetness and smell of urine. We are reminded of a neglected lady who has been with a VVF for ten years almost always hiding from her own community from the stigmata of wetness and smell of urine, long abandoned by her husband. She came to hospital only when something hard and solid "grew" out of her vagina! A urinary bladder stone protruding from her vagina was unbearable and she came to hospital where a successful sigma rectal pouch was constructed. It was impossible for her to find the right words to describe her happiness when she found herself dry and able again to reintegrate into the social norms of her community.

Similarly, the physical belonging, social belonging and community belonging were adversely affected by the conditions of our patients. Life at home, workplace, and school was affected. Following the sigma rectum pouch surgery, children were able to start schooling and follow instruction better in schools. Adults were able to maintain their employment and income and both adults and children were able to intimate with others freely.

In the sub domain of "becoming" this reconstructive surgery enabled the afflicted to achieve personal goals, hopes and aspirations.

It is clear that further research needs to be conducted in this area in order to reveal the association between sigma rectum pouch interventions and quality of life changes. It suffices to say at this juncture that human perceptions, expectations, and behaviour are controlled by influences extending far beyond surgery, clinical medicine and management. Quality of life therefore, is adapted to the lives of all humans, at any time, and from their individual perspectives.

The study of quality of life requires special structured questionnaires to have meaningful interpretation. In this series we did not use this type of structured questionnaires, we are therefore reporting on the results on quality of life with this limitation in mind. With respect to quality of life, most patients reported that bowel motions consisted of only urine and mucus, and that urine was mixed with faeces during one or two evacuations per day. Some patients reported that they could hold the rectal contents for more than six hours during the daytime and were discouraged to do so to prevent over distension of the reservoir; they were also advised to empty the reservoir at least once at night. All our patients were very reluctant to have urinary diversion methods with abdominal skin stoma and requiring external appliances, which had had various negative impacts on their psychosocial lives. They all refused to have a continent pouch with a catheterisable skin stoma. None of our patients, male or female had to change their dressing habits or diet pattern on account of Mainz Pouch II urinary diversion. All our adult patients were able to continue with their normal daily activities, furthermore in bed at night, all the patients were able to turn over freely without fear of sudden rectal soiling.

In this series of patients, there were 21 children with exstrophy epispadias complex requiring urinary diversion. Some of these children were late to start schooling because of their medical condition. All the school age children in this series were able to attend schooling normally after Mainz Pouch II surgery.

\section{DISCUSSION}

The objectives of urinary diversion are expanding from merely preserving kidney functions to enabling the patient to have a good quality of life while maintaining an acceptable dignity and social esteem (7). It is for these reasons that we as caregivers and our patients choose a method of diversion which does not involve external appliances. Wet cutaneous stomas are generally unacceptable to most of our patients because appliances are not easily available and even when available might be too expensive to our rural patients (8-10). In addition, to our younger and physically active exstrophy epispadia complex patients the psychological drawbacks of non-continent skin stoma in young adolescents cannot be overlooked. One other major advantage of Mainz Pouch II was that patients can freely turn over during sleep, whereas in patients with external urinary appliances cannot do the same.

Urinary incontinence does not represent a major psychosocial issue until the child enters school, as the parents and home provide a protective and understanding environment. With increasing social contact in school this issue becomes crucial; and with the onset of puberty, the body image becomes of much concern and quality of life issues associated with urinary diversion are becoming increasingly important 
to both patient and physician. Twenty five per cent of patients in this series had exstrophy epispadias complex as the indication of urinary diversion and therefore it is justifiable to describe the surgical goals in this congenital condition affecting children.

The management of sphincteric urinary incontinence in exstrophy epispadias complex is a challenging urological problem. The main treatment goals are to achieve complete urinary continence while preserving renal functions and achieving adequate external genitalia. Exstrophy epispadias complex often requires staged surgical approach with primary bladder closure as soon as possible after birth; subsequent bladder neck reconstruction and bilateral ureteroneocystostomy are undertaken when bladder capacity is adequate and in association with augmentation cystoplasty when bladder capacity is small. This approach results in satisfactory urinary continence in $32 \%$ to $80 \%$ of the patients, although further procedures are sometimes necessary to achieve the desired continence; the rate of re-operation can be as high as $75 \%$. For these disadvantages associated with primary bladder closure, we have changed our treatment strategy in bladder exstrophy epispadias complex patients since 1993 with primary urinary diversion to Mainz Pouch II and we have achieved satisfactory result.

Although the postoperative mortality and morbidity have remarkably decreased, the incidence of delayed complications of pyelonephritis and hyperchloremic acidosis, as well as night-time incontinence, remained significantly high. Recently, it became evident that most of these complications are essentially due to the unfavorable high-pressure conditions within the intact sigmoid colon and rectum. Functional studies proved that the pressure waves of physiologic bowel contractions toward the anus reach pressures exceeding $100 \mathrm{~cm} \mathrm{H} 2 \mathrm{O}$, and during defecation pressure in the intact sigmoid colon can reach up to $200 \mathrm{~cm} \mathrm{H2O}$. Detubularisation and reconfiguration of the sigmoid colon, rectum, or both eliminate the mass contractions and high-pressure peaks and improve their dynamics to function as a compliant low-pressure reservoir. A low-pressure reservoir for urine is created by antimesenteric splitting and side-to-side anastomosis of the rectosigmoid, the expectation being to obtain better continence rates and better protection of the upper tract than are achievable by ureterosigmoidostomy (11). Since the introduction of Mainz Pouch II to our institution in 1993, we have found it the procedure of choice to our community where it is highly accepted. Even among male patients, voiding while squatting did not affect their overall acceptance as they have learned to adapt to life as normally as possible.

Early complications at $13.2 \%$ (Table 2) were seen in this series of patients for instance; prolonged paralytic ileus was seen in only one patient, and this is comparable to other studies. Eight point four per cent patients developed superficial wound sepsis which was managed conservatively, however 1.2\% patient developed wound dehiscence requiring secondary suturing, and this is also comparable to other studies. Similarly, the $2.4 \%$ peri-operative deaths amongst the patients who had malignant disease is comparable to other series $(11,12)$. We also observed the development $3.6 \%$ mucoceles among male patients with exstrophy after cystectomy and genitalplasty due to retention of prostatic secretions in bladder and urethral remnant tissues and this phenomenon required secondary surgery to remove the tissues at the same time to reconstruct communication with the external urethral meatus. Pyelonephritis was seen only in $1.2 \%$ of patient sand this is on the lower side as some series have reported rates as high as $12 \%$. Mainz Pouch II diversion therefore shows good upper urinary tract protection. There was deterioration of renal function observed in $2.4 \%$ of patients and $3.6 \%$ unilateral mild to moderate hydronephrosis, non requiring surgical intervention which was comparable to other series. In this series of study, $1.2 \%$ of the patients developed stenosis of the uretero-sigmoid anastomosis during the follow-up period and this is on the lower side compared to that observed by Fisch et al (11) at $6.8 \%$. The once feared hyperchloremic acidosis can be prevented by regular acid status control and the administration of oral alkalising drugs. In this series, $3.6 \%$ of the patients developed metabolic acidosis of which $2.4 \%$, required oral sodium bicarbonate supplements. In comparison to other studies there was no serious acidosis in the present study as other studies reported need for alkalising drugs as high as $51 \%$ to $65 \%$. The need for bicarbonate supplementation is sometimes attributable to preoperative renal function and thus correct patient selection is important.

Incontinence after continent urinary diversion is devastating for patients and caregivers alike, in this series; the anal sphincter competence saline 
enema test was done as part of preoperatively workup in the adult patients. The high rate of continence in this study at 100\% daytime and $96.4 \%$ night time is quite satisfactory. The overall total continence rate achieved at $96.4 \%$ is comparable to other studies reporting $95.5 \%$ to $98.6 \%$ (11). There was however $3.6 \%$ patients who had occasional night time incontinence from the failed VVF repair group and we think that they might have had a suboptimal anal sphincter competence after obstetric trauma during their difficult delivery. The role of physiotherapy in this group of failed VVF repair patients was not tested in this series. The increased risk of night leak might also be caused by increased neural stimulation at night $(11,13)$.

The appearance of secondary malignancy is a well known complication after ureterointestinal anastomosis (14). This fact is important to those patient groups where a long life expectancy is expected such as exstrophy epispadias but might not be important in elderly patients undergoing cystectomy for carcinoma of the urinary bladder.

In conclusion, Mainz Pouch II reconstructive surgery is a safe and reproducible method of urinary diversion and serves as a satisfying method of continent urinary diversion in all age groups. Our results in this series are favorable and are comparable with results cited in other series elsewhere.

Mainz Pouch II reconstruction enabled the afflicted to achieve their personal goals, hopes and aspirations. It is clear that further research needs to be conducted in this area in order to reveal the association between sigma rectum pouch interventions and quality of life score changes.

It suffices to say at this juncture that human perceptions, expectations, and behaviour are controlled by influences extending far beyond surgery, clinical medicine and management. Quality of life therefore, is adapted to the lives of all humans, at any time: and from their individual perspectives (15).

The follow up show low complication rate with good results in terms of continence and quality of life, however, long term results remain to be evaluated.

\section{ACKNOWLEDGEMENTS}

The authors would like to thank the administration of Kilimanjaro Christian Medical Centre for allowing us the use of the hospital medical records and we would also like to thank our colleagues in the urology department whose support was important in the production of this paper.

\section{REFERENCES}

1. Coffey, R.C. Physiologic implantation of the severed ureter or common bile duct into the intestine. J. Amer. Med. Assoc. 1911; 56: 397-403.

2. Simon, J. Ectopia vesicae (absence of the anterior abdominal walls of the bladder and the pubis abdominal parietes); operation for directing the orifices of the ureters into the rectum; temporary success; subsequent death; autopsy. Lancet. 1852; 2: 568-570.

3. Stamey, T.A. The pathogenesis and complications of the electrolyte imbalance in ureterosigmoidostomy. Surg. Gynec. Obst. 1956; 103: 736-758.

4. Fisch, M., Wammack, R., Muller, S.C. et al. The Mainz Pouch II (sigma rectum pouch). J. Urol. 1993; 149: 258-263.

5. Spence, H.M., Hoffmann, W.W. and Fosmire, G.P. Tumours of the colon as a late complication of ureterosigmoidostomy for extrophy of the bladder. Brit. J. Urol. 1979; 51: 466.

6. Gittes, R.F. Carcinogenesis in ureterosigmoidostomy. Urol. Clin. North Amer. 1986; 13: 201.

7. Bjerre, B.D., Johansen, C. and Steven, K. Healthrelated quality of life after urinary diversion: continent diversion with the Kock pouch compared with ileal conduit; a questionnaire study. Scand. J. Urol. Nephrol. (Suppl). 1994; 157: 113.

8. Aboud, M.M., Eshleman, J.L., Mteta, K.A., et al. An 18-year review of types of continent urinary diversion applicable to the lesser-developed regions of Africa. East E Central Afr. J. Surg. 1999; 5:

9. Mteta, K.A., Mbwambo, J.S., Eshleman, J.L., et al. Urinary diversion in children with exstrophy epispadius. Alternative to primary bladder closure. Cent. Afr. J. Med. 2000; 46: 318-320.

10. Aboud, M.M. Early outcome of Mainz Pouch II urinary diversion. East \& Central Afr. J. Surg. 2001; 2:

11. Fisch, M., Wammack, R. and Hohenfellner, R. The sigma rectum pouch (Mainz pouch II). World J. Urol. 1996; 14: 68-72.

12. Bastian, P.J., Albers, P., Haferkamp A., et al. Modified ureterosigmoidostomy (Mainz Pouch II) in different age groups with different techniques of ureteric reimplantation. Brit. J. Surg. 2004; 94: 345-349.

13. Krogh, P. and Christian J. A study of the physiological variation in annal manometry. Brit. J. Surg. 1989; 76: 69-71.

14. Stein, R., Fisch, M., Stockle, M. and Hohenfellner, R. Urinary diversion in bladder exstrophy and incontinent epispadias: 25 years of experience. J. Urol. 1995; 154: 1177.

15. Testa, M.A. and Simonson, D.C. Assessment-of-quality of life. New Engl. J. Med. 1996; 334: 835-840. 